\title{
Spin polarization-scaling quantum maps and channels
}

\author{
Sergey N. Filippov ${ }^{1,2,3}$ and Kamil Yu. Magadov ${ }^{1, *}$ \\ ${ }^{1}$ Moscow Institute of Physics and Technology, Institutskii Per. 9, Dolgoprudny, Moscow Region 141700, Russia \\ ${ }^{2}$ Valiev Institute of Physics and Technology of Russian Academy of Sciences, Nakhimovskii Pr. 34, Moscow 117218, Russia \\ ${ }^{3}$ Steklov Mathematical Institute of Russian Academy of Sciences, Gubkina St. 8, Moscow 119991, Russia
}

\begin{abstract}
We introduce a spin polarization-scaling map for spin- $j$ particles, whose physical meaning is the decrease of spin polarization along three mutually orthogonal axes. We find conditions on three scaling parameters under which the map is positive, completely positive, entanglement breaking, 2-tensor-stable positive, and 2-locally entanglement annihilating. The results are specified for maps on spin-1 particles. The difference from the case of spin- $\frac{1}{2}$ particles is emphasized.
\end{abstract}

Keywords: Spin polarization, qubit, qutrit, positive map, quantum channel, entanglement breaking, 2-tensorstable properties

\section{INTRODUCTION}

Quantum states of a spin- $j$ particle are described by $(2 j+1) \times(2 j+1)$ density matrices $\varrho \in \mathcal{S}(\mathcal{H})$ satisfying the properties $\varrho^{\dagger}=\varrho, \operatorname{tr}[\varrho]=1$, and $\langle\varphi|\varrho| \varphi\rangle \geqslant 0$ for all $|\varphi\rangle \in \mathcal{H}$, $\operatorname{dim} \mathcal{H}=2 j+1$. Taking into account the normalization condition, the density matrix $\varrho$ is defined by $(2 j+1)^{2}-1$ real parameters, which are usually treated as components of the generalized Bloch vector [1, 2, 10, 19, 28. However, many physical phenomena can be explained and visualized via a spin polarization vector $\mathbf{p} \in \mathbb{R}$ with components $p_{i}=\operatorname{tr}\left[\varrho J_{i}\right]$, where $J_{1}, J_{2}, J_{3}$ are usual $(2 j+1)$-dimensional representations of angular momentum operators (see, e.g., 34). Angular momentum operators are Hermitian and satisfy the commutation relation $\left[J_{k}, J_{l}\right]=i e_{k l m} J_{m}$, where $e_{k l m}$ is the conventional Levi-Civita symbol and the summation over $m$ being assumed. Note that the spin-polarization vector p does not contain the full information about the quantum state if $j \geqslant 1$. Despite this fact, it is of great use in quantum physics and chemistry as its components represent average spin projections onto three orthogonal axes and are experimentally measurable. Linear transformations of the spin polarization vector include rotations and scaling. Rotations are attributed to the unitary evolution, so we do not consider them in the present paper. Physically motivated scaling of the spin polarization vector is described by a map $\Phi: \mathcal{B}(\mathcal{H}) \mapsto \mathcal{B}(\mathcal{H})$ of the following form:

$\Phi[X]=\frac{1}{2 j+1} \operatorname{tr}[X] I+\frac{3}{j(j+1)(2 j+1)} \sum_{i=1}^{3} \lambda_{i} \operatorname{tr}\left[X J_{i}\right] J_{i}$,

where $I \in \mathcal{B}(\mathcal{H})$ is the identity operator and $\lambda_{i} \in \mathbb{R}$. The factors take into account that $\operatorname{tr}[I]=2 j+1$ and $\operatorname{tr}\left[J_{k} J_{l}\right]=\frac{1}{3} j(j+1)(2 j+1) \delta_{k l}, \quad \delta_{k l}$ is the Kronecker delta. The map (1) is trace-preserving and unital, i.e. $\operatorname{tr}[\Phi[X]]=\operatorname{tr}[X]$ and $\Phi[I]=I$. Note that the map (1) differs in general from other classes of unital maps [21, 25]. Physical meaning of Eq. (1) is the transformation of the spin polarization

$$
p_{i} \mapsto \lambda_{i} p_{i}, \quad i=1,2,3 .
$$

\footnotetext{
*Deceased July 6, 2017.
}

In case of spin- $\frac{1}{2}$ particles, formula (1) transforms into a well-known Pauli qubit map $\Phi[X]=$ $\frac{1}{2}\left(\operatorname{tr}[X] I+\sum_{i=1}^{3} \lambda_{i} \operatorname{tr}\left[X \sigma_{i}\right] \sigma_{i}\right)$, where $\left(\sigma_{1}, \sigma_{2}, \sigma_{3}\right)$ is the conventional set of Pauli matrices (see, e.g., [11, 26]). The qubit $\left(j=\frac{1}{2}\right)$ map $\Phi$ is known to be positive if and only if $\left|\lambda_{i}\right| \leqslant 1$, completely positive if and only if $1 \pm \lambda_{3} \geqslant\left|\lambda_{1} \pm \lambda_{2}\right|$, entanglement breaking if and only if $\left|\lambda_{1}\right|+\left|\lambda_{2}\right|+\left|\lambda_{3}\right| \leqslant 1,2$-local-entanglement-annihilating if and only if $\lambda_{1}^{2}+\lambda_{2}^{2}+\lambda_{3}^{2} \leqslant 1$, 2-tensor-stable positive if and only if $1 \pm \lambda_{3}^{2} \geqslant\left|\lambda_{1}^{2} \pm \lambda_{2}^{2}\right|$ [4, 5, 7]. Similar characterization for higher spins $(j \geqslant 1)$ is still missing, so the goal of the present paper is to analyze analogous properties of such maps and illustrate them for qutrits $(j=1)$.

The paper is organized as follows. In Sec. III we analyze positivity of the map (1). In Sec. III, the criterion of complete positivity of such a map is presented. In Sec. IV] the entanglement breaking property is partially characterized. In Sec. V, we review the positivity and entanglement annihilation behaviour of the map $\Phi \otimes \Phi$. In Sec. VI] brief conclusions are presented.

\section{POSITIVITY}

We will refer to an operator $R$ as positive-semidefinite and write $R \geqslant 0$ if $\langle\varphi|R| \varphi\rangle \geqslant 0$ for all $|\varphi\rangle \in \mathcal{H}$. A map $\Phi$ is called positive if $\Phi[X] \geqslant 0$ for all $X \geqslant 0[33$.

Let us now analyze positivity of the spin polarizationscaling map (1).

Since each $J_{i}$ is a spin projection operator with eigenvalues $j, j-1, \ldots,-j$, eigenvalues of the operator $a_{1} J_{1}+a_{2} J_{2}+a_{3} J_{3}$ are $|\mathbf{a}|\{j, j-1, \ldots,-j\}$, where $|\mathbf{a}|=\sqrt{a_{1}^{2}+a_{2}^{2}+a_{3}^{2}}$. Therefore, the minimal eigenvalue of $\Phi[X]$ reads

$$
\frac{1}{2 j+1}\left(\operatorname{tr}[X]-\frac{3}{j+1} \sqrt{\sum_{i=1}^{3}\left(\lambda_{i} \operatorname{tr}\left[X J_{i}\right]\right)^{2}}\right) .
$$

Suppose $X \geqslant 0$. As $\left|\operatorname{tr}\left[X J_{i}\right]\right| \leqslant j \operatorname{tr}[X]$, the minimal value of (3) is non-negative if $1-\frac{3 j}{j+1} \sqrt{\sum_{i=1}^{3} \lambda_{i}^{2}} \geqslant 0$. Thus, we have found sufficient condition for positivity of the map $\Phi$.

Proposition 1. Spin-polarization-scaling map $\Phi$ is positive if $\sum_{i=1}^{3} \lambda_{i}^{2} \leqslant\left(\frac{j+1}{3 j}\right)^{2}$. 
The necessary condition for positivity of the map (1) follows from the particular form of the positive-semidefinite operator

$X=x_{0} I+\sum_{i=1}^{3} x_{i} J_{i}, \quad x_{0} \geqslant 0, \quad x_{i} \in \mathbb{R}, \quad x_{0} \geqslant j \sqrt{\sum_{i=1}^{3} x_{i}^{2}}$.

In fact, if $X$ is given by formula (4), then $\Phi[X]=$ $x_{0} I+\sum_{i=1}^{3} \lambda_{i} x_{i} J_{i}$ and $\Phi[X] \geqslant 0$ if and only if $x_{0} \geqslant$ $j \sqrt{\sum_{i=1}^{3} \lambda_{i}^{2} x_{i}^{2}}$. Suppose $x_{0}=j x_{1}$ and $x_{2}=x_{3}=0$, then $\Phi[X] \geqslant 0$ if $\left|\lambda_{1}\right| \leqslant 1$. Similarly, necessary conditions $\left|\lambda_{2}\right| \leqslant 1$ and $\left|\lambda_{3}\right| \leqslant 1$ appear for choices $x_{0}=j x_{2}$ $x_{1}=x_{3}=0$ and $x_{0}=j x_{3}, x_{1}=x_{2}=0$, respectively.

Proposition 2. Suppose the spin polarization-scaling map $\Phi$ is positive, then $\left|\lambda_{i}\right| \leqslant 1, i=1,2,3$.

\section{COMPLETE POSITIVITY}

A linear map $\Phi$ is called completely positive if $\Phi \otimes \operatorname{Id}_{k}$ is positive for all $k=1,2, \ldots$. Here $\operatorname{Id}_{k}$ is the identity transformation of $k$-dimensional operators $\mathcal{B}\left(\mathcal{H}_{k}\right)$.

Proposition 3. The spin polarization-scaling map $\Phi$ is completely positive if and only if

$I \otimes I+\frac{3}{j(j+1)}\left(\lambda_{1} J_{1} \otimes J_{1}-\lambda_{2} J_{2} \otimes J_{2}+\lambda_{3} J_{3} \otimes J_{3}\right) \geqslant 0$.

Proof. A linear map $\Phi: \mathcal{B}\left(\mathcal{H}_{d}\right) \mapsto \mathcal{B}\left(\mathcal{H}_{d}\right)$ is known to be completely positive if and only if its Choi matrix $\Omega_{\Phi}=\left(\Phi \otimes \operatorname{Id}_{d}\right)\left[\left|\psi_{+}\right\rangle\left\langle\psi_{+}\right|\right]$is positive-semidefinite [3] (see also [17, 18, 22, 29]), where $\left|\psi_{+}\right\rangle=\frac{1}{\sqrt{d}} \sum_{i=1}^{d}|i\rangle \otimes|i\rangle$ is the maximally entangled state and $\{|i\rangle\}_{i=1}^{d}$ is some orthonormal basis in $\mathcal{H}_{d}$.

For our construction of the Choi operator let us choose eigenvectors of the operator $J_{3}$ as the basis, namely, $J_{3}|j m\rangle=m|j m\rangle$ and $\left\langle j m^{\prime} \mid j m\right\rangle=\delta_{m^{\prime} m}, d=2 j+1$. Introduce auxiliary operators $J_{ \pm}=J_{1} \pm i J_{2}$, then $J_{ \pm}|j m\rangle=$ $\sqrt{(j \mp 1)(j \pm m+1)}|j m \pm 1\rangle$. Some algebra yields

$$
\begin{aligned}
& (2 j+1)^{2}(\Phi \otimes \mathrm{Id})\left[\left|\psi_{+}\right\rangle\left\langle\psi_{+}\right|\right] \\
= & \sum_{m, m^{\prime}=-j}^{j} \Phi\left[|j m\rangle\left\langle j m^{\prime}\right|\right] \otimes|j m\rangle\left\langle j m^{\prime}\right| \\
= & \sum_{m=-j}^{j}\left(I+\frac{3 m}{j(j+1)} \lambda_{3} J_{3}\right) \otimes|j m\rangle\langle j m| \\
& +\sum_{m=-j}^{j} \frac{3}{2 j(j+1)} \sqrt{(j-m)(j+m+1)} \\
& +\sum_{m=-j}^{j} \frac{3}{2 j(j+1)} \sqrt{\left(\lambda_{1} J_{1}-i \lambda_{2} J_{2}\right) \otimes|m\rangle\langle m+1|} \\
& \times\left(\lambda_{1} J_{1}+i \lambda_{2} J_{2}\right) \otimes|m\rangle\langle m-1| \\
= & I \otimes I+\frac{3}{j(j+1)}\left(\lambda_{1} J_{1} \otimes J_{1}-\lambda_{2} J_{2} \otimes J_{2}+\lambda_{3} J_{3} \otimes J_{3}\right) .
\end{aligned}
$$

Thus, $\Phi$ is completely positive if and only if the operator (6) is positive-semidefinite.

Example 1. If $j=\frac{1}{2}$, then angular momentum operators are given by matrices

$J_{1}=\frac{1}{2}\left(\begin{array}{ll}0 & 1 \\ 1 & 0\end{array}\right), \quad J_{2}=\frac{1}{2}\left(\begin{array}{cc}0 & -i \\ i & 0\end{array}\right), \quad J_{3}=\frac{1}{2}\left(\begin{array}{cc}1 & 0 \\ 0 & -1\end{array}\right)$

in the basis $\left\{\left|\frac{1}{2} \frac{1}{2}\right\rangle,\left|\frac{1}{2}-\frac{1}{2}\right\rangle\right\}$. The condition (5) reduces to $1 \pm \lambda_{3} \geqslant\left|\lambda_{1} \pm \lambda_{2}\right|$. Geometrically, these inequalities correspond a tetrahedron with vertices $(1,1,1)$, $(1,-1,-1),(-1,1,-1)$, and $(-1,-1,1)$ in the parameter space $\left(\lambda_{1}, \lambda_{2}, \lambda_{3}\right)$ 31.

Example 2. If $j=1$, then angular momentum operators are given by matrices

$$
\begin{aligned}
& J_{1}=\frac{1}{\sqrt{2}}\left(\begin{array}{ccc}
0 & 1 & 0 \\
1 & 0 & 1 \\
0 & 1 & 0
\end{array}\right), \quad J_{2}=\frac{1}{\sqrt{2}}\left(\begin{array}{ccc}
0 & -i & 0 \\
i & 0 & -i \\
0 & i & 0
\end{array}\right) \\
& J_{3}=\left(\begin{array}{ccc}
1 & 0 & 0 \\
0 & 0 & 0 \\
0 & 0 & -1
\end{array}\right)
\end{aligned}
$$

in the basis $\{|11\rangle,|10\rangle,|1-1\rangle\}$. The condition (5) reduces to $4-9\left(\lambda_{1}^{2}+\lambda_{2}^{2}+\lambda_{3}^{2}\right)+27 \lambda_{1} \lambda_{2} \lambda_{3} \geqslant 0,\left|\lambda_{i}\right| \leqslant \frac{2}{3}, i=1,2,3$. Geometrical figure corresponding to such inequalities is depicted in Fig. 11(a).

\section{ENTANGLEMENT BREAKING}

A positive-semidefinite operator $R \in \mathcal{B}\left(\mathcal{H}_{1}\right) \otimes \mathcal{B}\left(\mathcal{H}_{2}\right)$ is called separable (non-entangled) if there exist positivesemidefinite operators $R_{1}^{(k)}$ and $R_{2}^{(k)}$ such that $R=$ $\sum_{k} R_{1}^{(k)} \otimes R_{2}^{(k)}$ [15, 35]. A linear map $\Phi: \mathcal{H}_{1} \mapsto \mathcal{H}_{1}$ is called entanglement breaking if $(\Phi \otimes \mathrm{Id})[\varrho]$ is separable for all $\varrho \in \mathcal{S}\left(\mathcal{H}_{1} \otimes \mathcal{H}_{2}\right)$ and identity transformation Id : $\mathcal{H}_{2} \mapsto \mathcal{H}_{2}$ [12, 16, 20, 30, 32, The well-known result is that $\Phi$ is entanglement breaking if and only if the Choi matrix $\Omega_{\Phi}$ is separable.

The necessary condition for separability of $\Omega_{\Phi}$ is that $\Omega_{\Phi}^{\Gamma} \geqslant 0$, where $X^{\Gamma}=\sum_{j, j^{\prime}} I \otimes\left|j^{\prime}\right\rangle\left\langle j|X I \otimes| j^{\prime}\right\rangle\langle j|$ is the partially transposed operator, $X \in \mathcal{B}\left(\mathcal{H}_{1}\right) \otimes \mathcal{B}\left(\mathcal{H}_{2}\right)[13$ 27. Applying such a condition to the Choi matrix (6) and taking into account that in conventional basis $\{j m\}_{m=-j}^{J}$ the matrices $J_{x}^{\top}=J_{x}, J_{y}^{\top}=-J_{y}, J_{z}^{\top}=J_{z}$, we obtain the following result.

Proposition 4. Suppose the spin polarization-scaling map $\Phi$ is completely positive and entanglement breaking, then

$I \otimes I+\frac{3}{j(j+1)}\left(\lambda_{1} J_{1} \otimes J_{1} \pm \lambda_{2} J_{2} \otimes J_{2}+\lambda_{3} J_{3} \otimes J_{3}\right) \geqslant 0$.

Note that the requirement (9) is sufficient for the channel $\Phi$ to be entanglement binding [14].

Example 3. If $j=\frac{1}{2}$, then Eq. (9) is equivalent to $\left|\lambda_{1}\right|+$ $\left|\lambda_{2}\right|+\left|\lambda_{3}\right| \leqslant 1$

Example 4. If $j=1$, then Eq. (9) is equivalent to $4-$ $9\left(\lambda_{1}^{2}+\lambda_{2}^{2}+\lambda_{3}^{2}\right) \pm 27 \lambda_{1} \lambda_{2} \lambda_{3} \geqslant 0$. 
(a)

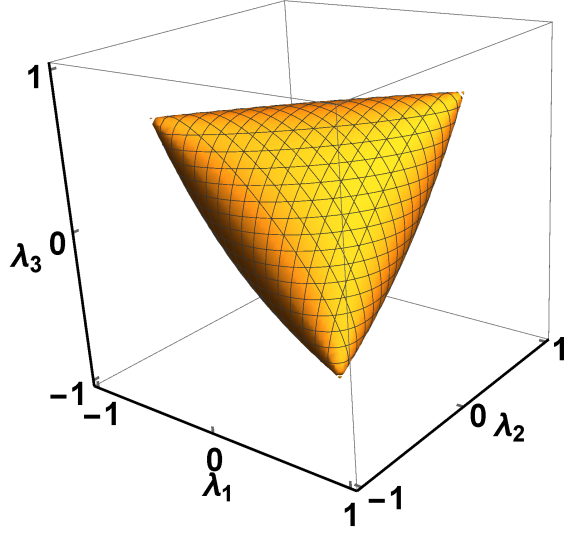

(b)

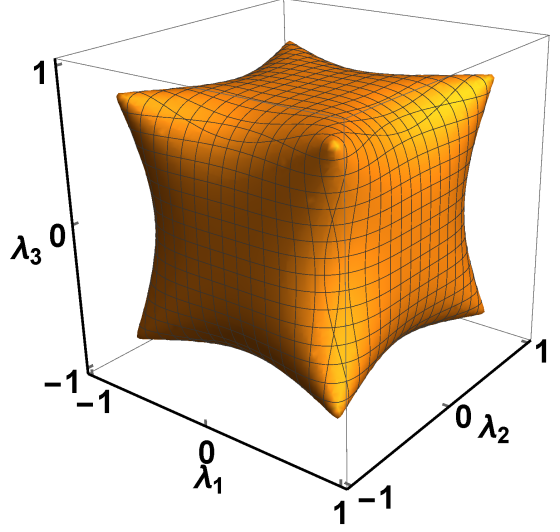

FIG. 1: Region of parameters $\left(\lambda_{1}, \lambda_{2}, \lambda_{3}\right)$ for which the spin-1 polarization map $\Phi$ is completely positive (a), the map $\Phi^{2}$ is completely positive (b).

\section{2-TENSOR-STABLE PROPERTIES}

Some properties of linear maps do not change under tensoring the map with itself, for instance, $\Phi \otimes \Phi$ is completely positive if and only if $\Phi$ is completely positive. Similarly, $\Phi \otimes \Phi$ is entanglement breaking if and only if $\Phi$ is entanglement breaking (see, e.g., 6]). However, other properties of a map can change drastically under tensor power. For example, the map $\Phi \otimes \Phi$ can be non-positive even if $\Phi$ is positive [5].

A linear map $\Phi$ is called 2-tensor-stable positive if $\Phi \otimes \Phi$ is positive [24].

Example 5. It is shown in Ref. 5] that the spin polarization-scaling map $\Phi$ given by Eq. (1) for qubits $\left(j=\frac{1}{2}\right)$ is 2-tensor-stable positive if and only if $\Phi^{2}$ is completely positive, i.e. $1 \pm \lambda_{3}^{2} \geqslant\left|\lambda_{1}^{2} \pm \lambda_{2}^{2}\right|$.

For higher spins $(j \geqslant 1)$ the result of Example 5 can be extended as follows.

Proposition 5. If the spin polarization-scaling map $\Phi$ is 2-tensor-stable positive, then $\Phi^{2}$ is completely positive.

Proof. Consider a positive-semidefinite operator $\left|\psi_{+}\right\rangle\left\langle\psi_{+}\right|$, where $\left|\psi_{+}\right\rangle=(2 j+1)^{-1 / 2} \sum_{m=-j}^{j}|j m\rangle \otimes|j m\rangle$. The action of the positive map $\Phi \otimes \Phi$ on such an operator reads

$$
\begin{aligned}
0 \leqslant(2 j+1)^{2}(\Phi \otimes \Phi)\left[\left|\psi_{+}\right\rangle\left\langle\psi_{+}\right|\right] \\
=(2 j+1)^{2}(\mathrm{Id} \otimes \Phi) \circ(\Phi \otimes \mathrm{Id})\left[\left|\psi_{+}\right\rangle\left\langle\psi_{+}\right|\right] \\
=(\operatorname{Id} \otimes \Phi)\left[I \otimes I+\frac{3}{j(j+1)}\right. \\
\left.\quad \times\left(\lambda_{1} J_{1} \otimes J_{1}-\lambda_{2} J_{2} \otimes J_{2}+\lambda_{3} J_{3} \otimes J_{3}\right)\right] \\
=I \otimes I+\frac{3}{j(j+1)}\left(\lambda_{1}^{2} J_{1} \otimes J_{1}-\lambda_{2}^{2} J_{2} \otimes J_{2}+\lambda_{3}^{2} J_{3} \otimes J_{3}\right) \\
=(2 j+1)^{2}\left(\Phi^{2} \otimes \mathrm{Id}\right)\left[\left|\psi_{+}\right\rangle\left\langle\psi_{+}\right|\right],
\end{aligned}
$$

i.e. the Choi matrix $\Omega_{\Phi^{2}}$ is positive-semidefinite and $\Phi^{2}$ is completely positive.
In contrast to the case $j=\frac{1}{2}$, for higher spins $(j \geqslant 1)$ Proposition 5 provides the necessary condition only. For instance, it is not hard to see that for $j=1$ there exists a spin polarization-scaling map $\Phi$ such that $\Phi^{2}$ is completely positive but $(\Phi \otimes \Phi)[|\varphi\rangle\langle\varphi|] \ngtr 0$ for the Schmidt-rank-2 state $|\varphi\rangle=\frac{1}{\sqrt{2}}(|11\rangle+|1-1\rangle)$.

In the case $j=1$, the map $\Phi^{2}$ is completely positive if and only if $4-9\left(\lambda_{1}^{4}+\lambda_{2}^{4}+\lambda_{3}^{4}\right)+27 \lambda_{1}^{2} \lambda_{2}^{2} \lambda_{3}^{2} \geqslant 0$, which is depicted in Fig. 1(b).

A linear map $\Phi: \mathcal{B}(\mathcal{H}) \mapsto \mathcal{B}(\mathcal{H})$ is called 2-locally entanglement annihilating [7 9, 23] if $(\Phi \otimes \Phi)[|\psi\rangle\langle\psi|]$ is separable for all $|\psi\rangle \in \mathcal{H} \otimes \mathcal{H}$. The same line of reasoning as for 2-tensor-stable positive maps leads to the following result.

Proposition 6. If the spin polarization-scaling map $\Phi$ is 2-locally entanglement annihilating, then $\Phi^{2}$ is entanglement breaking.

\section{CONCLUSIONS}

We have considered the physically motivated sets of operator maps for spin systems. The physical meaning of such maps is the degradation of spin polarization with scaling parameters $\lambda_{1}, \lambda_{2}, \lambda_{3}$ along the axes $x, y, z$, respectively. We have found conditions (necessary, or sufficient, or both) under which the spin polarization-scaling map is positive, completely positive, entanglement breaking, 2-tensor-stable positive, 2-locally entanglement annihilating. These results can be of use in the analysis of data, where only spin polarization degrees of freedom are available. The crucial difference between the cases of spin- $\frac{1}{2}$ and spin-1 particles is illustrated in a series of examples.

\section{Acknowledgments}

The study is supported by Russian Science Foundation under project No. 16-11-00084. 
[1] M. S. Byrd, C. A. Bishop, and Y.-C. Ou, General opensystem quantum evolution in terms of affine maps of the polarization vector, Phys. Rev. A 83, 012301 (2011).

[2] A. Chȩcińska and K. Wydkiewicz, Complete positivity conditions for quantum qutrit channels, Phys. Rev. A 80, $032322(2009)$.

[3] M.-D. Choi, Completely positive linear maps on complex matrices, Linear Algebra Appl. 10, 285 (1975).

[4] S. N. Filippov, PPT-Inducing, distillation-prohibiting, and entanglement-binding quantum channels, J. Russ. Laser Res. 35, 484 (2014).

[5] S. N. Filippov and K. Yu. Magadov, J. Phys. A: Math. Theor. 50, 055301 (2017).

[6] S. N. Filippov, A. A. Melnikov, and M. Ziman, Dissociation and annihilation of multipartite entanglement structure in dissipative quantum dynamics, Phys. Rev. A 88, 062328 (2013).

[7] S. N. Filippov, T. Rybár, and M. Ziman, Local twoqubit entanglement-annihilating channels, Phys. Rev. A 85, 012303 (2012).

[8] S. N. Filippov and M. Ziman, Bipartite entanglementannihilating maps: Necessary and sufficient conditions, Phys. Rev. A 88, 032316 (2013).

[9] S. N. Filippov and M. Ziman, Entanglement sensitivity to signal attenuation and amplification, Phys. Rev. A 90, 010301(R) (2014).

[10] S. K. Goyal, B. N. Simon, R. Singh, and S. Simon, Geometry of the generalized Bloch sphere for qutrits, J. Phys. A: Math. Theor. 49, 165203 (2016).

[11] T. Heinosaari and M. Ziman, The Mathematical Language of Quantum Theory, (Cambridge University Press, Cambridge, 2012).

[12] A. S. Holevo, Quantum coding theorems, Russ. Math. Surveys 53, 1295 (1998)

[13] M. Horodecki, P. Horodecki, and R. Horodecki, Separability of mixed states: necessary and sufficient conditions, Phys. Lett. A 223, 1 (1996).

[14] P. Horodecki, M. Horodecki, R. Horodecki, Binding entanglement channels, J. Mod. Opt. 47, 347 (2000).

[15] R. Horodecki, P. Horodecki, M. Horodecki, and K. Horodecki, Quantum entanglement, Rev. Mod. Phys. 81, 865 (2009)

[16] M. Horodecki, P. W. Shor, and M. B. Ruskai, Entanglement breaking channels, Rev. Math. Phys. 15, 629 (2003).

[17] A. Jamiołkowski, Linear transformations which preserve trace and positive semidefiniteness of operators, Rep. Math. Phys. 3, 275 (1972).

[18] M. Jiang, S. Luo, and S. Fu, Channel-state duality, Phys.
Rev. A 87, 022310 (2013).

[19] V. Karimipour, A. Mani, and L. Memarzadeh, Characterization of qutrit channels in terms of their covariance and symmetry properties, Phys. Rev. A 84, 012321 (2011).

[20] C. King, Maximization of capacity and $l_{p}$ norms for some product channels, J. Math. Phys. 43, 1247 (2002).

[21] L. J. Landau and R. F. Streater, On Birkhoff's theorem for doubly stochastic completely positive maps of matrix algebras, Linear Algebra and its Applications 193, 107 (1993).

[22] W. A. Majewski and T. I. Tylec, Comment on Channelstate duality, Phys. Rev. A 88, 026301 (2013).

[23] L. Moravčíková and M. Ziman, Entanglement-annihilating and entanglement-breaking channels, J. Phys. A: Math. Theor. 43, 275306 (2010).

[24] A. Müller-Hermes, D. Reeb, and M. M. Wolf, Positivity of linear maps under tensor powers, J. Math. Phys. 57, 015202 (2016).

[25] M. Nathanson and M. B. Ruskai, Pauli diagonal channels constant on axes, J. Phys. A: Math. Theor. 40, 8171 (2007).

[26] M. A. Nielsen and I. L. Chuang, Quantum Computation and Quantum Information, (Cambridge University Press, Cambridge, 2000).

[27] A. Peres, Separability criterion for density matrices, Phys. Rev. Lett. 77, 1413 (1996).

[28] D. Petz and H. Ohno, Generalizations of Pauli channels, Acta Math. Hungar. 124, 165 (2009).

[29] J. de Pillis, Linear transformations which preserve Hermitian and positive semidefinite operators, Pacific J. of Math. 23, 129 (1967).

[30] M. B. Ruskai, Qubit entanglement breaking channels, Rev. Math. Phys. 15, 643 (2003).

[31] M. B. Ruskai, S. Szarek, and E. Werner, An analysis of completely-positive trace-preserving maps on $\mathrm{M}_{2}$, Linear Algebra Appl. 347, 159 (2002).

[32] P. W. Shor, Additivity of the classical capacity of entanglement-breaking quantum channels, J. Math. Phys. 43, 4334 (2002).

[33] E. Størmer, Positive linear maps of operator algebras, Acta Math. 110, 233 (1963).

[34] D. A. Varshalovich, A. N. Moskalev, and V. K. Khersonskii, Theory of Angular Momentum, (World Scientific, Singapore, 1988).

[35] R. F. Werner, Quantum states with Einstein-PodolskyRosen correlations admitting a hidden-variable model, Phys. Rev. A 40, 4277 (1989). 